GLASNIK MATEMATIČKI

Vol. 39(59)(2004), $341-348$

\title{
A NOTE ON PERMEABILITY FOR A NETWORK OF THIN CHANNELS
}

\author{
SANJA MARUŠIĆ \\ University of Zagreb, Croatia
}

\begin{abstract}
We study a viscous flow through a periodic network of thin channels with small period $\varepsilon$ and small thickness $\varepsilon \delta$. We consider two cases $\lim _{\varepsilon \rightarrow 0}\left(\lim _{\delta \rightarrow 0}\right)$ and $\lim _{\delta \rightarrow 0}\left(\lim _{\varepsilon \rightarrow 0}\right)$. In both cases the limit (homogenized) problem is the Darcy law with the same permeability tensor.
\end{abstract}

\section{INTRODUCTION}

It is well known that a laminar newtonian flow through a porous medium obeys the Darcy law (see e.g. [11]). It is also known that the Darcy permeability tensor depends on complicated geometry of pores in an implicit way. In this paper we give an extensive study of the permeability tensor and the Darcy pressure in case of very simple porous media consisting of a periodic net of thin channels.

The problem has two small parameters, the period of a network $\varepsilon$ and the thickness of channels $\varepsilon \delta$. We prove that the limit pressure, as both $\varepsilon$ and $\delta$ tend to zero, does not depend on order in which the two limits are taken. In both cases $\lim _{\delta \rightarrow 0} \lim _{\varepsilon \rightarrow 0}, \lim _{\varepsilon \rightarrow 0} \lim _{\delta \rightarrow 0}$ the limit pressure satisfies the Darcy problem (2.12), (2.13).

We define the geometry by periodic repetition of the cell $Y_{\varepsilon}^{\delta}$. For simplicity of this presentation we consider two-dimensional situation and we suppose that the fluid part of the unit cell consists of two perpendicular channels ${ }^{1}$. Thus the rescaled cell $\left.Y^{\delta} \subset Y, Y=\right]-1 / 2,1 / 2\left[{ }^{2}\right.$ consists of 2 channels

2000 Mathematics Subject Classification. 76S05, 35B27, 35B25, 35B40.

Key words and phrases. Periodic porous media, network of thin channels, commuting limits.

${ }^{1}$ That assumption is not essential for our results. The only important thing is that we have only one junction in each cell. 
with thickness $\delta$ intersected in one junction point denoted $\mathrm{O}$ as on figure 1. Each channel has a profile $\left.\delta S^{i}, S^{i}=\right]-a^{i}, a^{i}\left[, a^{i}>0\right.$ and direction $\mathbf{e}^{i} \in \mathbf{R}^{2}, \mathbf{e}^{i} \cdot \mathbf{e}^{j}=\delta_{i j}, i=1,2$, i.e.

$$
\begin{aligned}
& P_{\delta}^{1}=\left\{y=\left(y_{1}, y_{2}\right) \in Y ; y_{2} \in \delta S^{1}\right\} \\
& P_{\delta}^{2}=\left\{y=\left(y_{1}, y_{2}\right) \in Y ; y_{1} \in \delta S^{2}\right\} .
\end{aligned}
$$

Now $Y^{\delta}=\cup_{j=1}^{2} P_{\delta}^{j}$ and $Y_{\varepsilon}^{\delta}=\varepsilon Y^{\delta}$.

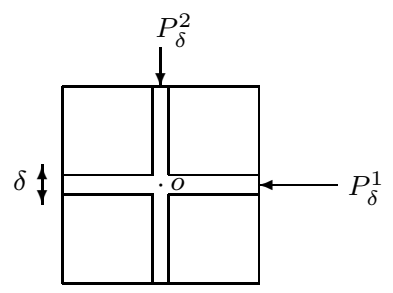

Figure 1. Periodicity cell $Y^{\delta}$

To get an $\varepsilon$-periodic medium we define a periodic lattice

$$
E_{\delta}^{\varepsilon}=\varepsilon \bigcup_{\alpha \in \mathbf{Z}^{2}}\left(\alpha+Y^{\delta}\right) .
$$

We chose a locally Lipschitz bounded domain $\Omega \subset \mathbf{R}^{2}$, and intersect it with $E_{\delta}^{\varepsilon}$ to define the fluid domain

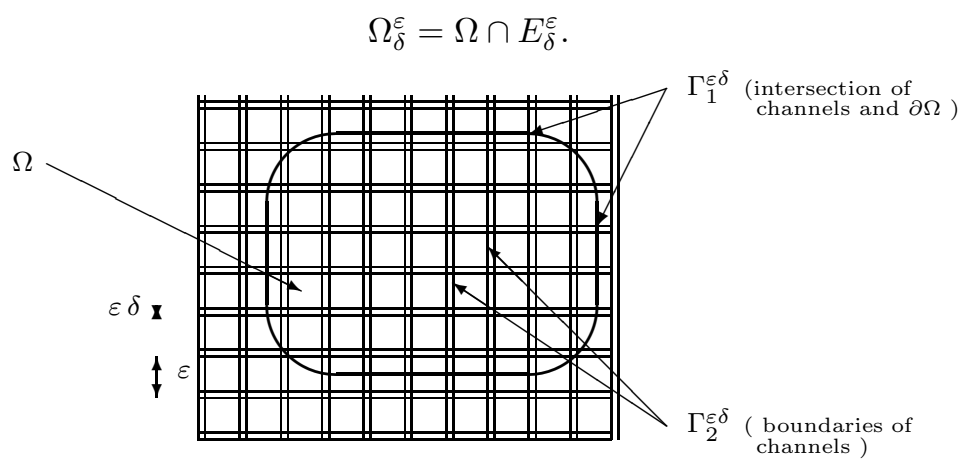

FiguRE 2. $\varepsilon$-periodic network of thin channels intersected by $\Omega$

The microscopic problem is linearized for the sake of simplicity and we have chosen the mixed boundary conditions: no-slip on boundary of pores and pressure condition on exterior boundary. It reads

$$
\begin{aligned}
& -\mu \Delta u^{\varepsilon \delta}+\nabla p^{\varepsilon \delta}=0, \quad \operatorname{div} u^{\varepsilon \delta}=0 \text { in } \Omega_{\delta}^{\varepsilon} \\
& p^{\varepsilon \delta}=q, u^{\varepsilon \delta} \times \mathbf{n}=0 \text { on } \Gamma_{1}^{\varepsilon \delta}, u^{\varepsilon}=0 \text { on } \Gamma_{2}^{\varepsilon \delta},
\end{aligned}
$$


where $\Gamma_{1}^{\varepsilon \delta}=E_{\delta}^{\varepsilon} \cap \partial \Omega, \Gamma_{2}^{\varepsilon \delta}=\partial E_{\delta}^{\varepsilon} \cap \Omega$ and $\mathbf{n}$ is the unit exterior normal on $\Gamma_{1}^{\varepsilon \delta}$.

Such problem is uniquely solvable due to its linearity (see [8]).

Our goal is to study the asymptotic behavior of the flow as $\varepsilon$ and $\delta$ tend to zero. The limit $\lim _{\delta \rightarrow 0} \lim _{\varepsilon \rightarrow 0}$ was found in our previous paper [7]. Here, we find the commuted limit $\lim _{\varepsilon \rightarrow 0} \lim _{\delta \rightarrow 0}$ and compare it with $\lim _{\delta \rightarrow 0} \lim _{\varepsilon \rightarrow 0}$. In fact the network approximation $\lim _{\delta \rightarrow 0}$ was obtained in [6] and we here find its limit as the mesh size $\varepsilon \rightarrow 0$. It turns out that the two double limits are equal, in the sense that the limit $\lim _{\varepsilon \rightarrow 0} \lim _{\delta \rightarrow 0}$ can be seen as a finite difference discretization of the Darcy problem obtained as $\lim _{\delta \rightarrow 0} \lim _{\varepsilon \rightarrow 0}$. Thus the two limits commute.

Homogenization of reticulated structures was first studied by Bakhvalov and Panasenko (see book [1] and the references therein). Further exstensive study, with results similar to ours, but for elasticity, was done by Cioranescu and Saint Jean Paulin (see, e.g. book [5] and the references cited). An alternative approach to the study of thin, mesh-structure domains, in case when $\varepsilon$ and $\delta$ are related, via two-scale convergence with respect to concentrated measures, was developed by Bouchitté G., Fragalà I. in [4] and applied for a double porosity model derivation in Bourgeat A., Chechkin G., Piatnitski A. [3]. In that method $\varepsilon$ is proportional to $\delta$ and it allows to study simultaneously processes in both, thin mash and surrounding media.

\section{CASE $\lim _{\varepsilon \rightarrow 0} \lim _{\delta \rightarrow 0}$}

In this case we first use the method of homogenization to pass to the limit as $\varepsilon \rightarrow 0$. Regardless of our special structure of the unit cell, the following result can be proved (following [12], with slight modifications due to the pressure boundary condition on $\Gamma$ ):

Theorem 1. Let $\left(v^{\delta}, p^{\delta}\right)$ be the solution of the Darcy system

$$
\begin{aligned}
& v^{\delta}=-\mathbf{K}^{\delta} \nabla p^{\delta} \quad \text { in } \Omega \\
& \operatorname{div} v^{\delta}=0 \text { in } \Omega \\
& p^{\delta}=q \text { on } \Gamma .
\end{aligned}
$$

Then there exists an extension of $\left(u^{\varepsilon \delta}, p^{\varepsilon \delta}\right)$ to whole $\Omega$, denoted by the same symbol, such that

$$
\begin{aligned}
& p^{\varepsilon \delta} \rightarrow p^{\delta} \quad \text { strongly in } L^{2}(\Omega) \\
& \varepsilon^{-2} u^{\varepsilon \delta} \rightarrow v^{\delta} \quad \text { weakly in } L^{2}(\Omega)^{2}
\end{aligned}
$$


as $\varepsilon \rightarrow 0$. The permeability tensor $\mathbf{K}^{\delta}$ is computed from an auxiliary problem posed in $Y^{\delta}$

$$
\begin{aligned}
& -\mu \Delta w_{\xi}^{\delta}+\nabla_{y} \pi_{\xi}^{\delta}=\xi \text { in } Y^{\delta} \\
& \operatorname{div}_{y} w_{\xi}^{\delta}=0 \text { in } Y^{\delta} \\
& \left(w_{\xi}^{\delta}, \pi_{\xi}^{\delta}\right) \text { is } Y^{\delta}-\text { periodic } \\
& w_{\xi}^{\delta}=0 \text { on } S^{\delta}
\end{aligned}
$$

where $S^{\delta}=\partial Y^{\delta} \backslash \partial Y$, by

$$
\mathbf{K}^{\delta} \xi=\int_{Y^{\delta}} w_{\xi}^{\delta} .
$$

Next we study the limit as $\delta \rightarrow 0$. Such problem was considered in [7] and an explicit formula for limiting permeability $\mathbf{K}^{0}$, as $\delta \rightarrow 0$ was found. An approximation of $\left(w_{\xi}^{\delta}, \pi_{\xi}^{\delta}\right)$ was sought in the separated form and, as expected, it turned out that the eigenvectors of $\mathbf{K}^{0}$ coincide with the axes of tubes forming the net in $Y^{\delta}$, i.e. with $\mathbf{e}^{i}$. More precisely, $\mathbf{K}^{0}$ is defined as follows.

Let $\mathbf{e}^{i}, i=1,2$ be the axis of 2 channels forming the cell $Y^{\delta}$ and let $\chi^{i}$ be the corresponding torsion functions, i.e. the solutions to the problems

$$
-\mu\left(\chi^{i}\right)^{\prime \prime}=1 \text { in } S^{i}, \chi^{i}=0 \text { for } \xi= \pm a^{i} \Rightarrow \chi^{i}(\xi)=\frac{1}{2 \mu}\left[\left(a^{i}\right)^{2}-\xi^{2}\right] .
$$

Then

$$
\mathbf{K}^{0} \xi=\sum_{i=1}^{2}\left(\xi \cdot \mathbf{e}^{i}\right)\left\langle\chi^{i}\right\rangle \mathbf{e}^{i}
$$

where

$$
\left\langle\chi^{i}\right\rangle=\int_{-a^{i}}^{a^{i}} \chi^{i}(\xi) d \xi=\frac{2}{3 \mu}\left(a^{i}\right)^{3} .
$$

It was proved in [7] that, for such approximation, the following result holds:

Theorem 2. There exists $C>0$, independent from $\delta$, such that

$$
\left|\delta^{-3} \mathbf{K}^{\delta}-\mathbf{K}^{0}\right| \leq C \delta^{1 / 2} .
$$

Furthermore $p^{\delta} \rightarrow p^{0}$ weakly in $H^{1}(\Omega)$, where

$$
\begin{aligned}
& \operatorname{div} \mathbf{K}^{0} \nabla p^{0}=0 \quad \text { in } \Omega \\
& p^{0}=q \text { on } \Gamma .
\end{aligned}
$$

Thus, the macroscopic (Darcy) pressure in case $\lim _{\delta \rightarrow 0} \lim _{\varepsilon \rightarrow 0}$ satisfies (2.12), $(2.13)$. 


\section{CASE $\lim _{\delta \rightarrow 0} \lim _{\varepsilon \rightarrow 0}$}

Here we start with the limit as $\delta \rightarrow 0$. This is a singular perturbation problem and it has been studied in [6] (see also [2] for the case of Dirichlet exterior boundary condition, for the velocity). It was found that the limit pressure in each junction point $T_{i j}^{\varepsilon}=\varepsilon(i, j)$ satisfies the Kirchhoff law. If all four neighboring nodes $T_{i \pm 1 j}^{\varepsilon}$ and $T_{i j \pm 1}^{\varepsilon}$ are inside the domain $\Omega$ then it reads

$$
\begin{gathered}
\left\langle\chi^{1}\right\rangle\left[2 p^{\varepsilon}(i, j)-p^{\varepsilon}(i-1, j)-p^{\varepsilon}(i+1, j)\right]+ \\
+\left\langle\chi^{2}\right\rangle\left[2 p^{\varepsilon}(i, j)-p^{\varepsilon}(i, j-1)-p^{\varepsilon}(i, j+1)\right]=0,
\end{gathered}
$$

where $p^{\varepsilon}(i, j)=p^{\varepsilon}\left(T_{i j}^{\varepsilon}\right)$.

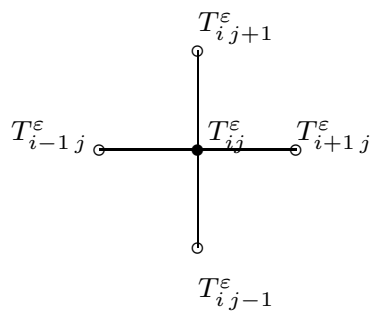

Figure 3. Points involved in the Kirchhoff formula

If some of the neighboring nodes, for instance $T_{i-1 j}^{\varepsilon}$ is out of the domain we replace it in the Kirchhoff law by the point where $\overline{T_{i-1 j}^{\varepsilon} T_{i j}^{\varepsilon}}$ cuts the boundary and we denote that point of intersection by $T_{i-1 j}^{*}$. In that point the value of the pressure is prescribed by the boundary condition, i.e.

$$
\begin{gathered}
p^{\varepsilon}(i-1, j)=q\left(T_{i-1 j}^{*}\right) \equiv q(i-1, j) . \\
\left\langle\chi^{1}\right\rangle\left[\frac{p^{\varepsilon}(i, j)-q(i-1, j)}{\operatorname{dist}\left(T_{i j}^{\varepsilon}, T_{i-1 j}^{*}\right)}+\frac{p^{\varepsilon}(i, j)-p^{\varepsilon}(i+1, j)}{\varepsilon}\right] \\
+\frac{\left\langle\chi^{2}\right\rangle}{\varepsilon}\left[2 p^{\varepsilon}(i, j)-p^{\varepsilon}(i, j-1)-p^{\varepsilon}(i, j+1)\right]=0 . \\
\partial \Omega \longrightarrow T_{T_{i-1 j}^{\varepsilon} \circ T_{i-1 j}^{*}}^{T_{i j-1}^{\varepsilon}}
\end{gathered}
$$

FiguRe 4. Near the boundary 
The pressure $p^{\varepsilon}$ is now defined by the Kirchhoff law only in the junction points. In order to estimate the difference $p^{\varepsilon \delta}-p^{\varepsilon}$ we need to extend it to the whole $\Omega_{\delta}^{\varepsilon}$. In [6] it was done in a complicated way by constructing the interior layer corrector in vicinity of the junction. Here we do not need such precision, and we extend $p^{\varepsilon}$ by constant on the intersection of two channels forming $Y_{\varepsilon}^{\delta}(i, j)=\varepsilon\left[(i, j)+Y_{\varepsilon}^{\delta}\right]$, i.e.

$$
p^{\varepsilon}(x)=p^{\varepsilon}(i, j) \quad \text { for } x \in B_{\delta}^{\varepsilon}(i, j),
$$

where $B_{\delta}^{\varepsilon}(i, j)=\varepsilon\left(P_{\delta}^{1}(i, j) \cap P_{\delta}^{2}(i, j)\right)$ with $P_{\delta}^{k}(i, j)=(i, j)+P_{\delta}^{k}, k=1,2$. In the rest of $(i, j)$-th cell we interpolate as

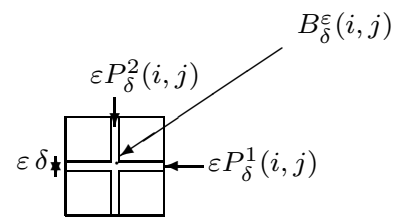

Figure 5. $(i, j)$-th cell $Y_{\varepsilon}^{\delta}(i, j)$

$$
\begin{array}{cl}
p^{\varepsilon}(x)=p^{\varepsilon}(i, j)+ & \frac{\left(x_{1}-\varepsilon\left(i+\delta a^{2}\right)\right)}{\varepsilon\left(1-\delta a^{2}\right)}\left[p^{\varepsilon}(i+1, j)-p^{\varepsilon}(i, j)\right] \\
\text { for } & \frac{x_{1}}{\varepsilon} \in\left[i+\delta a^{2}, i+1\right], \frac{x_{2}}{\varepsilon} \in j+\delta S^{2} \\
p^{\varepsilon}(x)=p^{\varepsilon}(i-1, j)+ & \frac{\left(x_{1}-\varepsilon(i-1)\right)}{\varepsilon\left(1-\delta a^{2}\right)}\left[p^{\varepsilon}(i, j)-p^{\varepsilon}(i-1, j)\right] \\
\text { for } & \frac{x_{1}}{\varepsilon} \in\left[i-1, i-\delta a^{2}\right], \frac{x_{2}}{\varepsilon} \in j+\delta S^{2} \\
p^{\varepsilon}(x)=p^{\varepsilon}(i, j)+ & \frac{\left(x_{2}-\varepsilon\left(j+\delta a^{1}\right)\right)}{\varepsilon\left(1-\delta a^{1}\right)}\left[p^{\varepsilon}(i, j+1)-p^{\varepsilon}(i, j)\right] \\
\text { for } & \frac{x_{2}}{\varepsilon} \in\left[j+\delta a^{1}, j+1\right], \frac{x_{1}}{\varepsilon} \in i+\delta S^{1} \\
p^{\varepsilon}(x)=p^{\varepsilon}(i, j-1)+ & \frac{\left(x_{2}-\varepsilon(j-1)\right)}{\varepsilon\left(1-\delta a^{1}\right)}\left[p^{\varepsilon}(i, j)-p^{\varepsilon}(i, j-1)\right] \\
\text { for } & \frac{x_{2}}{\varepsilon} \in\left[j-1, j-\delta a^{1}\right], \frac{x_{1}}{\varepsilon} \in i+\delta S^{1}
\end{array}
$$

For such approximation we have the following estimate:

TheOREm 3. Let $p^{\varepsilon \delta}$ be the microscopic pressure defined by the Stokes system (1.1), (1.2) and let $p^{\varepsilon}$ be the pressure defined by the Kirchhoff law and 
extended as in (3.3) and (3.4). Then there exists $C>0$, independent from $\delta$, such that

$$
\left|\Omega_{\delta}^{\varepsilon}\right|^{-1 / 2}\left|p^{\varepsilon \delta}-p^{\varepsilon}\right| \leq C \sqrt{\delta} .
$$

Proof. The following estimate was proved in [6] :

$$
\left|\Omega_{\delta}^{\varepsilon}\right|^{-1 / 2}\left|p^{\varepsilon \delta}-p^{\varepsilon}\right|_{L^{2}\left(\Omega_{\delta}^{\varepsilon} \backslash B_{\delta}^{\varepsilon}\right)} \leq C[\delta \log (1 / \delta)]^{3 / 2},
$$

where $B_{\delta}^{\varepsilon}$ is the union of all junction regions where channels intersect $B_{\delta}^{\varepsilon}(i, j)$, i.e.

$$
B_{\delta}^{\varepsilon}=\left(\bigcup_{(i, j) \in \mathbf{Z}^{2}} B_{\delta}^{\varepsilon}(i, j)\right) \cap \Omega .
$$

On the other hand estimating the interior layer corrector from [6], we conclude

$$
\left|\Omega_{\delta}^{\varepsilon}\right|^{-1 / 2}\left|p^{\varepsilon \delta}\right|_{L^{2}\left(B_{\delta}^{\varepsilon}\right)} \leq C[\delta \log (1 / \delta)]^{3 / 2} .
$$

Finally

$$
\left|\Omega_{\delta}^{\varepsilon}\right|^{-1 / 2}\left|p^{\varepsilon}\right|_{L^{2}\left(B_{\delta}^{\varepsilon}\right)} \leq C \sqrt{\delta}
$$

Such approximation $p^{\varepsilon}$ is related with the Darcy problem $(2.12),(2.13)$ because (3.1) is exactly the finite difference discretization of (2.12) in interior points of the $\varepsilon$-mesh on $\Omega$, while (3.2) is exactly its discretization near the boundary, taking into account the boundary condition (2.13). Therefore, as $\varepsilon \rightarrow 0, p^{\varepsilon}$ converges to $p^{0}$, the solution of $(2.12),(2.13)$ in the sense that:

TheOREM 4. Let $\mathcal{G}^{\varepsilon}$ be the union of all the nodes (junction points), i.e.

$$
\mathcal{G}^{\varepsilon}=\{x \in \Omega ; x=(i \varepsilon, j \varepsilon), i, j \in \mathbf{Z}\}
$$

and let $\|w\|_{\varepsilon}=\max _{x \in \mathcal{G}^{\varepsilon}}|w(x)|$. Suppose that the solution $p^{0}$ of the Darcy problem (2.12), (2.13) belongs to $C^{4}(\bar{\Omega})$. Then there exists a constant $C>0$, independent on $\varepsilon$, such that

$$
\left\|p^{\varepsilon}-p^{0}\right\|_{\varepsilon} \leq C \varepsilon^{2} .
$$

For the proof see for instance Theorem IV. 7 in [9] or [10].

REMARK 1. If those two channels in the unit cell were not perpendicular the discretization of (2.12), (2.13) would be in nonorthogonal mesh and therefore untypical. However it would still be the finite difference discretization of the same operator.

Thus, we conclude that the limit $\lim _{\varepsilon \rightarrow 0} \lim _{\delta \rightarrow 0} p^{\varepsilon \delta}=p^{0}$, where $p^{0}$ is the solution of the Darcy problem (2.12), (2.13). 


\section{REFERENCES}

[1] N.S. Bakhvalov and G.P. Panasenko, Homogenisation: Averaging processes in periodic media. Mathematical problems in the mechanics of composite materials, Kluwer, 1989.

[2] F. Blanc, O. Gipouloux, G. Panasenko and A.M. Zine, Asymptotic analysis and partial asymptotic decomposition of domain for Stokes equation in tube structure, Math. Models Methods Appl. Sci. 9 (1999), 1351-1378.

[3] A. Bourgeat, G. Chechkin and A. Piatnitski, Singular double porosity model, Appl.Anal. 82 (2003), 103-116.

[4] G. Bouchitté and I. Fragalà, Homogenization of thin structures by two-scale method with respect to measure, SIAM J.Math.Anal. 32 (2001), 1198-1226.

[5] D. Cioranescu and J. Saint Jean Paulin, Homogenization of reticulated structures, Springer series in Applied Mathematical Sciences 136, Springer, New York, 1999.

[6] E. Marušić-Paloka, Rigorous justification of the Kirchhoff law for junction of thin pipes filled with viscous fluid, Asymptotic Anal. 33 (2003), 51-66.

[7] E. Marušić-Paloka and S. Marušić, Computation of the permeability tensor for a fluid flow through a periodic net of thin channels, Appl. Anal. 64 (1997), 27-37.

[8] C. Conca, F. Murat and O. Pironneau, The Stokes and Navier-Stokes equations with boundary conditions involving the pressure, Japan J.Math. 20 (1994), 263-318.

[9] A.A. Samarskii, Vvedenije v teorju raznostnih shem, Nauka, 1971.

[10] A.A. Samarskii, The theory of difference schemes, Marcel Dekker, 2001.

[11] E. Sanchez-Palencia, Nonhomogeneous media and vibration theory, Lecture Notes in Physics 127, Springer, Berlin-New York, 1980.

[12] L. Tartar, Convergence of the homogenization process, Appendix to [11].

S. Marušić

Faculty of Transport and Traffic Engineering

University of Zagreb

Vukelićeva 4, 10000 Zagreb

Croatia

E-mail: marusics@mafpz.fpz.hr

Received: 07.05.2003.

Revised: 10.12.2003. 